\title{
QUANTIFICATION OF RIGHT AND LEFT VENTRICULAR FUNCTION WITH REAL-TIME THREE-DIMENSIONAL ULTRASOUND
}

\author{
E. D. Angelini ${ }^{1}$, A. F. Laine ${ }^{1}$, J. Donis ${ }^{2}$, D. Gersony ${ }^{2}$, S. Homma ${ }^{2}$ \\ ${ }^{1}$ Department of Biomedical Engineering, Columbia University, New York, NY, USA \\ ${ }^{2}$ Department of Medicine, Columbia University, New York, NY, USA
}

\begin{abstract}
This paper presents a study for validation of real-time three-dimensional ultrasound as a diagnostic tool in patients with primary pulmonary hypertension. Dynamic analysis of the heart is performed via quantification of right and left ventricle volumes. Segmentation of ventricular cavities is performed via a deformable-model for a set of denoised frames spread over an entire cardiac cycle. Spatio-temporal denoising is carried out by brushlet analysis that has been optimized by incorporating space and time coherence in texture characterization. Quantitative measures for right and left ventricular ejection fraction are compared to clinical MRI of the same patients. Keywords - Segmentation, echocardiography, denoising, RV volume, $L V$ volume, brushlet.
\end{abstract}

\section{INTRODUCTION}

Quantification of ventricular volume from standard twodimensional echocardiography is commonly performed via planar measurements and use of a geometric model for volume estimation. This method is routinely applied in clinical practice for assessment of left ventricular (LV) volume and ejection fraction. The left ventricle has an ellipsoidal shape with strong symmetry along a central axis, which allows volume estimation from $2 \mathrm{D}$ echocardiographic images using geometrical models. On the other hand, the right ventricle is crescent shaped and does not show any axis of symmetry. For this reason, right ventricular volume cannot be estimated from planar views and its function is not well quantified during an echocardiographic examination. Some cardiac pathologies such as congenital heart disease and primary pulmonary hypertension (PPH) can result in an increase of the right ventricular volume and affect pumping function on the right side while, at least in the initial phase of the condition, the left ventricle volume and ejection fraction remain normal. In such a situation, assessment of right ventricular function would be useful for early diagnosis and accurate assessment of the severity of the pathology.

The recent introduction of real-time three-dimensional (RT3D) ultrasound can overcome limitations associated with planar and early generations of 3D ultrasound by acquiring an entire cardiac volume at once using a matrix phased array [1]. With this new probe, since no reconstruction is required, right and left cavities can be segmented directly from the acquired data. The main drawback of RT3D ultrasound is its very limited resolution in B-scan mode, the high level of speckle noise embedded in the data and the artifacts introduced by the 1:16 downsampling scheme for the (transmitter/receiver) volumetric scanning.

We have previously developed a denoising tool adapted to the true four-dimensional nature of RT3D ultrasound data. We have shown that a two-dimensional deformable model applied to denoised RT3D ultrasound can provide accurate LV volume measures in healthy patients [2]. This study presents some new insights on the spatio-temporal denoising scheme using multiscale brushlet analysis. We have optimized the denoising process by exploiting both time and space coherence. Extensive testing of analysis parameters was performed to optimize the contrast of the denoised data while preserving textural appearance of the myocardium.

This study also presents quantitative analysis of LV and RV function using two-dimensional deformable model segmentation on denoised RT3D ultrasound data. We compared ejection fraction measures to manual tracings on both RT3D ultrasound and MRI. The ultimate goal of this study is to construct patient-specific accurate dynamic models of the heart using RT3D ultrasound and validate this new modality for clinical echocardiographic screening.

\section{Methodology}

Quantification of ventricular volumes from RT3D ultrasound data included denoising via brushlet expansion in space and time and segmentation of the denoised data with a $2 \mathrm{D}$ deformable model [2]. Both denoising and segmentation were optimized from their original implementation to better accommodate the properties of RT3D ultrasound signal, as presented below.

\section{Spatio-temporal denoising of RT3D ultrasound}

When performing denoising, it is crucial to get reliable model of the nature of the noise. Backscattering of echo signals create interference patterns in the acquired signal called speckles. These speckles degrade the resolution of a signal and corrupt the specificity of gray level intensities. Fully developed speckle noise in envelope-detected ultrasound signals can be modeled by a Rayleigh distribution [3, 4]. The Rayleigh density function $p(\vec{X})$ of the envelope signal $\vec{X}$ is given by:

$$
p(\vec{X})=\frac{\vec{X}}{\sigma^{2}} e^{\frac{-\vec{X}^{2}}{2 \sigma^{2}}},
$$

where $\sigma^{2}$ is the backscattered energy of the echo signal. We have tested and validated this noise model on in-vitro phantom data acquired in a tank of water. Mapping of the Rayleigh distribution on slice histograms, via maximum likelihood estimation of the parameter $\sigma$ showed a very strong correlation between the model and the data. Once the Rayleigh model was validated for speckle noise distribution, the ultrasound signal could be further modeled as [5]:

$$
\vec{X}=\vec{f}+\sqrt{\vec{f}} * \vec{n},
$$

where $\vec{X}$ is the observation, $\vec{f}$ is the true signal and $\vec{n}$ is a random noise component with zero mean, uncorrelated with $\vec{f}$. Using this model we can approximate the variance of the noise component $\vec{n}$ in homogeneous areas via local statistics derived from the observation signal $\vec{X}$. The noise variance 
$\sigma_{n}^{2}$ is a parameter of the mean value of the signal $\vec{f}$ and is approximated at pixel $(i, j)$ by:

$$
\sigma_{n_{i, j}}^{2}=\sigma_{X_{i, j}}^{2} / \mu_{X_{i, j}}
$$

where $\mu_{X_{i, j}}$ and $\sigma_{X_{i, j}}^{2}$ are the local mean and variance of the observation signal $\vec{X}$ estimated at pixel $(i, j)$. From this model, the noise process $\vec{n}$ can be estimated as:

$$
\vec{n}=(\vec{X}-\vec{f}) / \sqrt{\vec{f}} .
$$

We tested this model on clinical RT3D ultrasound data. The signal component $\vec{f}$ was estimated via local averaging. The model of Equation (4) provided a good estimation of the random noise component $\vec{n}$ without discernable structural patterns. We also estimated the noise variance using Equation (3). These results are illustrated in Figure 1 below.

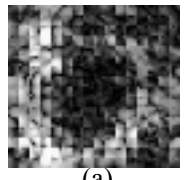

(a)

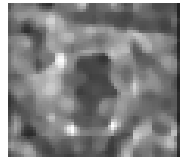

(b)

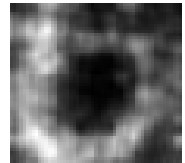

(c) (d)

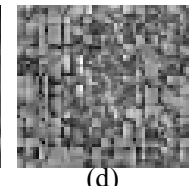

Fig. 1. Modelization of speckle noise component. (a) Original slice, (b) noise variance, (c) coherent energy process, (d) random noise component with no structural pattern.

With a reliable noise model, we were able to optimize brushlet denoising by adapting the thresholding level to noise variance. This methodology was inspired by the optimal minimax approach used in standard wavelet denoising procedures.

After performing empirical testing on RT3D clinical data, we concluded that minimax hard-thresholding performed better than non-adaptive hard and soft thresholding at removing speckle noise while enhancing myocardial walls. This resulted in denoised data with better contrast and excellent preservation of the original texture of cardiac muscle. An illustration of spatial denoising in 3D using fixed and adaptive hard thresholding is provided in Figure 2 below.

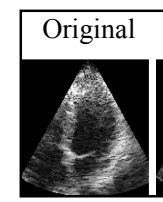

(a.1)

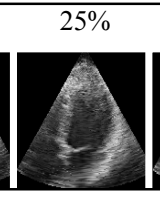

(a.2)

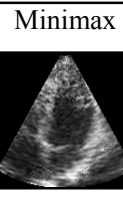

(a.3)

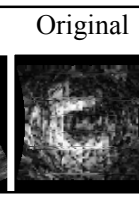

(b.1)

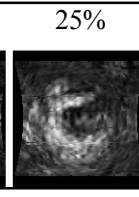

(b.2)

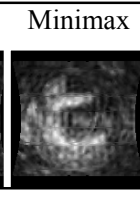

(b.3)
Fig. 2. Spatial denoising of RT3D ultrasound via hard thresholding (HT). (1) Original slice, (2) denoised slice via HT at $25 \%$ of coefficient maxima and (3) minimax HT are displayed in (a) long axis and (b) short axis views extracted from a clinical data set.

\section{Temporal denoising of RT3D ultrasound}

Temporal denoising is crucial for the localization of moving cardiac structures such as mitral valve and myocardial wall borders. Since time is involved in the deformation of the cardiac wall and in the physics of acquisition of the transducer, a best-adapted denoising protocol should include temporal analysis. With $(2 \mathrm{D}+\mathrm{T})$ or $(3 \mathrm{D}+\mathrm{T})$ ultrasound data, clinicians usually need to display $2 \mathrm{D}$ slices in very slow motion to make their final decision on the exact location of myocardial wall borders and small structures such as the mitral valve. Our brushlet denoising scheme uses similar temporal averaging approaches by incorporating spatial coherence in time to improve denoising performance, enhance moving structures while at the same time eliminate non-coherent noise.

Working with brushlet basis functions, we initially projected the four dimensional volume in the Fourier domain. We showed that including the temporal dimension improved denoising performance on both phantom and clinical data [6]. Processing the data as a four dimensional volume isolates dominant texture moving in time. We further extended temporal denoising by combining analysis of the time dimension in the Fourier and temporal domains. When projecting the time dimension on brushlet functions in the temporal domain, thresholding of the low amplitude coefficients isolates coherent mechanical movements of anatomical structures that can be expressed as a sum of complex exponentials. Such movements are analogous to harmonic pendulum oscillations. We have optimized the denoising procedure with phantom and clinical data and identified the following sequence which provided the best results: (1) temporal denoising in time domain, followed by (2) temporal denoising in Fourier domain. Both denoising procedures were again performed with four sub-quadrants in each dimension and thresholding of (1) all coefficients for temporal analysis and (2) lower frequency coefficients for frequency analysis. An illustration of temporal denoising in 4-D combining temporal and frequency analysis is shown in Figure 3 below.

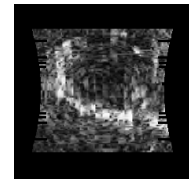

(a)

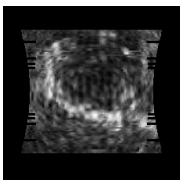

(b)

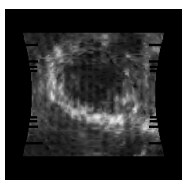

(c)

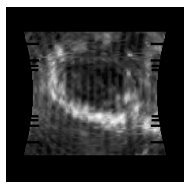

(d)
Fig. 3: Spatio-temporal denoising with brushlet analysis. (a) Original slice, (b) slice denoised in 3D space, (c) slice further denoised in time with temporal analysis, (d) slice further denoised in time with frequency analysis.

\section{Segmentation of RT3D ultrasound with $2 D$ deformable model}

Our initial deformable model implementation followed the work $\mathrm{Xu}$ et al. [7] using a finite difference scheme.

For each slice, the deformable model is initialized as a five pixels radius circle inside the cavity to segment. The position of the circles is manually initialized for every 10 slices and linearly interpolated for slices in between. Internal "balloon" forces expand the model along the normal direction while external forces, derived from the image gradient, stop the model at strong edges. However, with texturized images, it is very difficult to define strong edges from gradient information without stopping the model at false edges. To overcome this issue we had to modify the convergence criteria. Incorporating a priori knowledge is essential in the situation where several slices show only part of the myocardial wall so that no convergence of the model reflecting a true equilibrium of the forces can be attained. The model deforms under the influence of internal and external forces with associated weights defined empirically. The model will stop at its final position under two situations: either the average position of the set of points over 50 iterations varies below a certain threshold or the maximum number of iterations has been reached. The threshold value and the maximum number of iterations were also selected 
empirically. The maximum number of iterations was set in the range of 300-1000 depending on the cavity size. Nevertheless it is important to note here that we have identified structural parameters (i.e. elasticity, rigidity, viscosity) that performed robustly for the set of 10 patients reported in this study.

\section{RESULTS}

\section{Validation with $2 D$ Echo and MRI}

Volumes and ejection fraction were evaluated using RT3D ultrasound and MRI. MRI data were acquired either before or after the echocardiographic examination. Manual tracing was performed on both RT3D ultrasound and MRI as detailed below:

1) Manual tracing on RT3D data:

Typical RT3D volumes contained 300 slices in the long axis dimension with a slice thickness of $0.308 \mathrm{~mm}$. Between 10 and 17 frames were acquired per cardiac cycle. An expert cardiologist manually traced endocardial contours on selected slices and computed ventricular volumes via sector planedisks summation using a dedicated software package.

2) Manual tracing on MRI data:

MRI slices were $8 \mathrm{~mm}$ thick, without gaps, and were acquired at a rate of $40-50 \mathrm{~ms} /$ frame. An expert radiologist manually segmented the entire set of 10-15 slices available with a dedicated software package.

For both modalities, trabeculations and papillary muscles were excluded while moderator bands were included inside the cavity. Even though manual tracing was performed using the same anatomical definition of the ventricular cavity, many potential sources of error remain. MRI image quality is far superior to RT3D ultrasound but its spatial resolution is 26 times lower, leading to potential volume effect errors with MRI. Manual tracing of RT3D ultrasound was performed on long axis slices of the original data while selection of delimiting planes at the apex and the valve planes, for the deformable model segmentation, was performed by a different cardiologist on denoised short-axis slices. Right and left ventricular cavity segmentation was performed on different volumes for RT3D data but on the same volume for MRI. Taking into account these considerations the goal of this study will only focus on ejection fraction measures and will not compare volume measures directly. Ejection fraction (EF) was computed from end diastole volume (EDV) and end systole volumes (ESV) as:

$$
E F \%=100 \times \frac{(E D V-E S V)}{E D V} .
$$

\section{A. Right and left ventricular ejection fractions}

The ejection fraction measures were evaluated for right and left ventricular cavities on a set of 10 patients with primary pulmonary hypertension. To assess the agreement between the different methods, we performed a BlandAltman statistical analysis [8]. We considered measures from MRI data as the gold standard and computed errors of RT3D measures vs. MRI [9, 10]. Results of the Bland-Altman analysis are displayed in Figure 4.
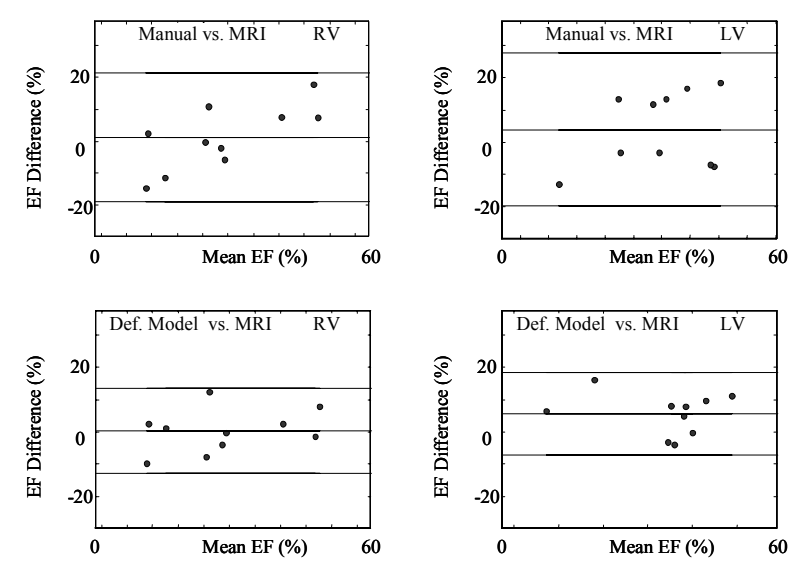

Fig. 4. Bland-Altman statistical analysis for LV and RV EF measures with manual tracing and deformable model (Def. Model) on RT3D ultrasound vs. manual tracing on MRI. Average EF difference and 95\% confidence interval are displaved on each plot.

\section{DISCUSSION}

This study focused on patients with primary pulmonary hypertension (PPH). This condition results in narrowing of the lung blood vessels that leads to increase pressure in the right ventricle and eventually heart failure. The cause of this disease is unknown, its real incidence difficult to evaluate and there are no treatments available to cure or halt its progression. Diagnosis of PPH is very delicate since preliminary symptoms such as chest pain and shortness of breath are not very specific. Because of the increase in RV pressure, the RV volume increases to produce more effort to expel blood through the constricted vessels. As the RV size increases, its shape becomes round while the LV cavity is compressed. These anatomical changes can be easily and accurately depicted with an echocardiographic exam. Quantification of RV and LV volumes and function can further characterize the stage of the disease and its severity. Analysis of the results showed that the deformable model applied to denoised RT3D ultrasound performed as accurately as manual tracing and reduced the $95 \%$ confidence interval by $34 \%$ for RV and $45 \%$ for $\mathrm{LV}$ volumes! Preliminary studies for RV volume measures with 3D ultrasound have reported inter- and intra- observer variability ranging from $4 \%$ to $9 \%$ [11]. One study for quantification of LV function with RT3D ultrasound reported inter-observer variability of $3.9 \%$ and intra observer variability of $2.4 \%$ for EF measure with sector plane-disks summation [9], as performed in this study. A similar study for RV measures with RT3D ultrasound showed an inter observer variability of $8.1 \%$ for stroke volume [2]. We previously presented results for comparison of manual tracing versus deformable model using RT3D ultrasound [12]. This study showed that the deformable model could achieve $8 \%$ accuracy when compared to MRI for quantification of LV volumes and ejection fraction. Observer variability should be taken into account when evaluating the performance of our segmentation method and comparing to manual tracing.

The set of ten patients used in this study presented great diversity in the quality of the data and the shapes and sizes of the ventricles. The quality of the RT3D ultrasound data depended on the acoustic window of the patients and on the ability of the cardiologist to acquire a good view of the 
patient's heart that included the entire cavity. In several cases, acquisition parameters had to be adapted to increase the depth setting and the field of view, sacrificing for acquisition quality. Because of the enlarged RV cavity, the left ventricle can become very small in some patients and its shape can become very distorted. The deformable model is well adapted in such situations as it can accommodate any shape convexity while ensuring continuity and smoothness of the contour. Volume ranges for this study were the following: RV ESV 50-191 ml, RV EDV 78-250 ml, LV ESV 12-49 ml, LV EDV 36-96 ml, RV EF 21-44\% and LV EF 41-68\% as obtained with manual tracing on RT3D. We illustrate the variety of RV and LV shapes we segmented in Figure 5 below for four long axis slices with the deformable model segmentation overlaid, and the segmented cavity reconstructed in 3D.

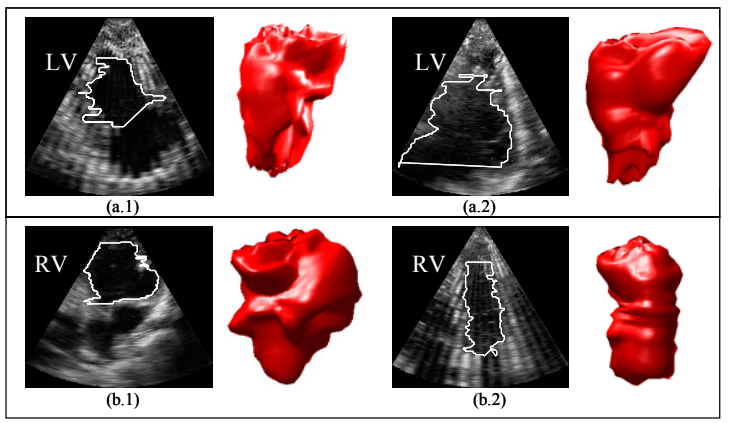

Fig. 5: LV and RV segmentation with deformable model performed on denoised RT3D ultrasound for two patients (1 and 2) at end diastole.

The ability of the deformable model to handle such complicated shapes in a robust fashion with the same set of intrinsic parameters is very encouraging. Nevertheless full automation of the segmentation process remains very challenging for two reasons. First, automatic initialization of the model is very difficult because of the presence of tissue inside the cavity such as moderator band. When initializing the model on top of such tissue, the model grows over it and includes it in the cavity,.while initializing next to the tissue makes the model stop at its border and can lead to part of the cavity not being included in the final segmentation. The second reason deals with the number of iterations, or more generally convergence criteria. As the ventricle size increases, the number of iterations required for the segmentation increases. But it is very delicate to increase the maximum number of iterations in every case since the septum wall that separates the two cavities very often shows low echo signal. In such situations, too many iterations can push the model to reach some equilibrium state and then further expand to pass over and include the adjacent cavity in the final segmentation. Potential improvements of the method include extension to $3 \mathrm{D}$ deformable surface $[13,14]$ to improve smoothness of the model in the third dimension and have a better control of the deformation constraints, multiscale approaches to speed up the segmentation by initializing with contours closer to the final solution and the use of automatic definition of structural parameters such as the medial axis as developed by Pizer and Stetten [12].

\section{CONCLUSION}

Quantification of RV and LV volumes with 2D ultrasound transducers is commonly performed via segmentation of the cavity on the planar view. Because of its crescent shape, RV volumes are never estimated from $2 \mathrm{D}$ images and can only be coarsely estimated with freehand and rotational 3D ultrasound. We have shown in this study that RT3D ultrasound can reconstruct and quantify accurately RV and LV ejection fraction in patients with primary pulmonary hypertension at various stage of severity. Potential applications include an improved diagnostic tool for assessment of PPH disease and better monitoring for drug treatment. This new RT3D ultrasound technology also offers the possibility of reconstructing RV and LV cavities in time for wall deformation analysis with patient specific dynamic models of the heart. Such application is of great importance for localization of ischemic tissue and septal wall defect and stress-echo echocardiography testing.

\section{ACKNOWLEDGMENT}

The authors thank Dr. Rola Saouaf from the Department of Radiology, Columbia University and our industrial partners, Volumetrics and Echotech 3D Imaging Systems.

\section{REFERENCES}

[1] G. Stetten, T. Ota, C. Ohazama, C. Fleishman, J. Castelucci, J. Oxaal, T. Ryan, J. Kisslo, and O. V. Ramm, "Real-time 3D ultrasound: A new look at the heart," Journal of Cardiovascular Diagnosis Procedures, vol. 15 , pp. 73-84, 1998.

[2] E. Angelini, A. Laine, S. Takuma, J. Holmes, and S. Homma, "LV volume quantification via spatio-temporal analysis of real-time 3D echocardiography," IEEE Transactions on Medical Imaging, in press.

[3] V. Dutt, "Statistical Analysis of Ultrasound Echo Envelope," in Ultrasound Research Laboratory. Rochester, MN: Mayo Foundation, 1995, pp. 181.

[4] T. Loupas, W. N. Mcdicken, and P. L. Allan, "An adaptive weighted median filtering for speckle suppression in medical ultrasonic images," IEEE Transactions on Circuits and Systems, vol. 36, pp. 129-135, 1989.

[5] X. Hao, S. Gao, and X. Gao, "A novel multiscale nonlinear thresholding method for ultrasonic speckle suppressing," IEEE Transactions on Medical Imaging, vol. 18, pp. 787-794, 1999.

[6] E. Angelini, S. Takuma, A. Laine, and S. Homma, "Spatio-temporal directional analysis of 4D echocardiography," Proceedings of SPIE45th Annual Meeting, San Diego, pp. 605-614, 2000.

[7] C. Xu and J. L. Prince, "Snakes, shapes and gradient vector Flow," IEEE Transactions on Image Processing, pp. 359-369, 1998.

[8] J. M. Bland and D. G. Altman, "Statistical methods for assessing agreement between two methods of clinical measurements," Lancet, vol. 1, pp. 307-310, 1986.

[9] T. Shiota, M. Jones, M. Chikada, C. Fleishman, J. Castellucci, B. Cotter, A. DeMaria, O. V. Ramm, J. Kisslo, T. Ryan, and D. Sahn, "Real-time three dimensional echocardiography for determining right ventricular stroke volume in an animal model of chronic right ventricular volume overload," Circulation, vol. 97, pp. 1897-1900, 1998.

[10] M. Vogel, M. Gutberlet, S. Dittrich, N. Hosten, and P. E. Lange, "Comparison of transthoracic three dimensional echocardiography with magnetic resonance imaging in the assessment of right ventricular volume and mass.," Heart, vol. 78, pp. 127-130, 1997.

[11] S. Takuma, T. Ota, T. Muro, I. Oropesa, R. Sciacca, L. Mendoza, M. R. D. Tullio, D. K. Blood, J. Yoshikawa, and S. Homma, "Assessment of left ventricular function by real-time $3 \mathrm{D}$ echocardiography compared with conventional noninvasive methods," Journal of the American Society of Echocardiography, vol. 14, pp. 275-284, 2001.

[12] G. D. Stetten and S. M. Pizer, "Medial-node models to identify and measure objects in real-time 3-D echocardiography," IEEE Transactions on Medical Imaging, vol. 18, pp. 1025-1034, 1999.

[13] X. Papademetris, A. J. Sinusas, D. P. Dione, and J. S. Duncan, "Estimation of 3D left ventricular deformation from echocardiography," Medical Image Analysis, vol. 5, pp. 17-28, 2001.

[14] J. Montagnat, H. Delingette, N.Scapel, and N. Ayache, "Surface simplex meshes for 3D medical image segmentation," Proceedings of IEEE International Conference on Robotics and Automation, pp. 864 - 870, 2000 . 